\title{
Técnica de microincisão na cirurgia do túnel do carpo
}

Luciano Ferreira de Holanda', Rafael Rodrigues Holanda², Amauri Pereira da Silva Filho², José da Costa Leão Segundoº, Vanessa Milanezi Holanda ${ }^{3}$, Benedito Jamilson Araújo Pereira ${ }^{4}$

Universidade Federal de Campina Grande, Hospital Antônio Targino e Clínica de Neurologia

e Neurocirurgia Domício Holanda, Campina Grande, PB, Brasil

\section{RESUMO}

Contexto: O tratamento proposto para síndrome do túnel do carpo é dividido em conservador e cirúrgico. O tratamento conservador versa em fisioterapia e uso de medicamentos. A cirurgia para liberação do túnel do carpo é um assunto corrente na literatura, motivo de diversas publicações, principalmente a partir dos anos 1950. Na liberação tradicional, o cirurgião faz uma incisão de $5 \mathrm{~cm}$ a $6 \mathrm{~cm}$, estendida longitudinalmente em relação à prega formada pelo punho, e realiza a liberação do ligamento transverso do carpo sob visualização direta. Objetivo: Apresentar a técnica cirúrgica desenvolvida por nós, que consiste em uma microincisão de $1 \mathrm{~cm}$ na linha média da prega do punho e recortar os bordos do retináculo dos flexores, e demonstrar as vantagens dessa técnica, perante a vigente. Casuística: A técnica foi utilizada em 40 pacientes de ambos os gêneros, das mais variadas faixas etárias, atendidose acompanhados no Hospital Antônio Targino e na Clínica de Neurologia e Neurocirurgia Domício Holanda, (ambos situados na cidade de Campina Grande, PB) com síndrome do túnel do carpo no período de janeiro de 1993 a dezembro de 2008. Resultados: Verificou-se que houve diminuição da dor, a técnica proporcionou melhor efeito estético, uma vez que, pela microincisão na linha da prega palmar, pouco tempo após o procedimento a cicatriz perdia-se entre as pregas palmares; em nenhum dos pacientes acompanhados após a cirurgia houve recidiva, constituindo uma vantagem sobre a técnica vigente; quanto à cicatrização, não houve formação de queloides em nenhum dos pacientes, ratificando-se melhor reposta cicatrizante; melhor exequibilidade ao cirurgião e menor tempo cirúrgico. Conclusão: A técnica apresentada oferece resultados extraordinários, diminuindo a chance de recidivas, trazendo melhor exequibilidade ao cirurgião e levando maior satisfação aos pacientes.

\section{PALAVRAS-CHAVE}

Síndrome do túnel do carpo, cirurgia.

\section{ABSTRACT}

Micro-incision for the surgery of carpal tunnel syndrome

Background: The proposed treatment for carpal tunnel syndrome is divided into conservative and surgical. Conservative treatment consists in physiotherapy and medication. Surgery to release the carpal tunnel is a current topic in the literature, subject to various publications, mostly from the $50 \mathrm{~s}$. In the traditional release, the surgeon makes an incision from $5 \mathrm{~cm}$ to $6 \mathrm{~cm}$ long, extended longitudinally in relation to the fold formed by the handle, and holds the release of the transverse carpal ligament under direct visualization. Objective: To present the surgical technique developed by us, which is a micro-incision $1 \mathrm{~cm}$ long in the midline of the wrist crease and cut the edges of the flexor retinaculum and demonstrate the advantages of this technique. Methods: The technique was used in forty patients presenting carpal tunnel syndrome, of both genders in a wide range of age groups from January 1993 to December 2008. Results: We found in all the cases that the pain was relieved, a better cosmetic result, the surgical scar was imperceptible shortly after the procedure lost in the palmar crease, there was no recurrence, providing an advantage over the usual techniques as for healing, no keloid formation confirming that there was better healing response, best feasibility for the surgeon and a shorter duration of surgery. Conclusion: The technique presented provides extraordinary results, reducing the chance of recurrence, easier feasibility to the surgeon and more patient's satisfaction.

\section{KEY WORDS}

Carpal tunnel syndrome, surgery.

1 Professor de Neurocirurgia e Neurologia da Universidade Federal de Campina Grande, PB.

2 Neurocirurgiões do Hospital Antônio Targino, Campina Grande, PB.

3 Médica residente em Neurocirurgia da equipe CENNA, SP.

4 Acadêmico de medicina da Universidade Federal de Campina Grande. 


\section{Introdução}

Assim como está descrita na literatura, observamos a limitação das atividades de indivíduo acometido pela síndrome do túnel do carpo (STC), o que traz prejuízos tanto para a vida do indivíduo, diminuindo sua autoestima, quanto para o setor produtivo, pois esse indivíduo não produz o máximo de sua capacidade, pela limitação que a dor provoca. Antes de tudo, o profissional de saúde habilitado que atende a esse paciente tem de indicar uma terapêutica que vá solucionar aquela sintomatologia. Caso o tratamento clínico, que sempre deve ter a prioridade, não traga os objetivos esperados, deve se partir para o tratamento cirúrgico.

No que diz respeito ao tratamento cirúrgico, vemos a evolução de técnicas nestes últimos 60 anos; todas elas trazem reformulações das anteriores com aperfeiçoamentos, mas vez por outra ainda deixavam sequelas, tais como uma cicatriz cirúrgica grande, uma cicatrização defeituosa e, o que é pior, às vezes com a recidiva do problema. Nossa expectativa é que a técnica desenvolvida por nós traga resolução para esses problemas, além de um pleno restabelecimento da saúde de nossos pacientes, o escopo do cotidiano do médico.

\section{Pacientes e métodos}

Quarenta pacientes de ambos os sexos, das mais variadas faixas etárias, atendidos e acompanhados no Hospital Antônio Targino e na Clínica de Neurologia e Neurocirurgia Domício Holanda (ambos situados na cidade de Campina Grande, PB) com STC decorrente de compressão do nervo mediano, participaram do estudo, no período de janeiro de 1993 a dezembro de 2008. Todos foram acompanhados pelo autor e por um estudante de medicina, que desconhecia a investigação clínica do primeiro.

Todos os pacientes obedeceram aos seguintes critérios de inclusão para participar do estudo: presença de dor ou parestesia nas regiões inervadas pelo nervo mediano e com resultados eletroneuromiográficos confirmando a compressão do nervo mediano e aceitação por livre e espontânea vontade de participar de nosso estudo. Foram excluídas da casuística as pessoas que não preenchiam o critério diagnóstico da síndrome, apresentavam fratura prévia e lesões duplas - como radiculopatia cervical e polineuropatia - e aquelas que não quiseram participar da pesquisa.
De cada paciente foram descritos o quadro clínico, os sinais encontrados ao exame físico e os resultados dos exames complementares. No exame clinico, foram pesquisados os sinais de Tinel-Hoffmann e Phalen, como sinais que acompanham a síndrome.

A técnica cirúrgica desenvolvida por nós consiste em uma microincisão de $1 \mathrm{~cm}$ na linha média da prega do punho, levantamento da pele por meio de pequenos afastadores Farabeufs, em seguida feita a divulsão dos planos até a completa visualização do retináculo dos flexores. Após a visualização, recortam-se os bordos do retináculo dos flexores para evitar a recidiva da síndrome.

\section{Resultados}

A pesquisa da validação da técnica foi feita acompanhando a evolução do paciente no pós-operatório no que tange dor, recidiva da síndrome e satisfação do paciente. Verificou-se que houve diminuição da dor, a técnica proporcionou melhor efeito estético, uma vez que, pela microincisão na linha da prega palmar, pouco tempo após o procedimento a cicatriz perdia-se entre as pregas palmares, o que sem sombra de dúvidas já se constitui em uma vantagem; em nenhum dos pacientes acompanhados após a cirurgia houve recidiva, constituindo uma vantagem sobre a técnica vigente; quanto à cicatrização, não houve formação de queloides em nenhum dos pacientes, ratificando-se melhor reposta cicatrizante; sem contar a melhor exequibilidade ao cirurgião, além de proporcionar menor tempo cirúrgico (ver sequência de figuras: figura 1, relativa à incisão convencional; figura 2, relativa à marcação de nossa incisão; figura 3, mostrando a sequência cirúrgica; figura 4 , mostrando o pós-operatório sequencial, atentar para a discreta cicatriz).

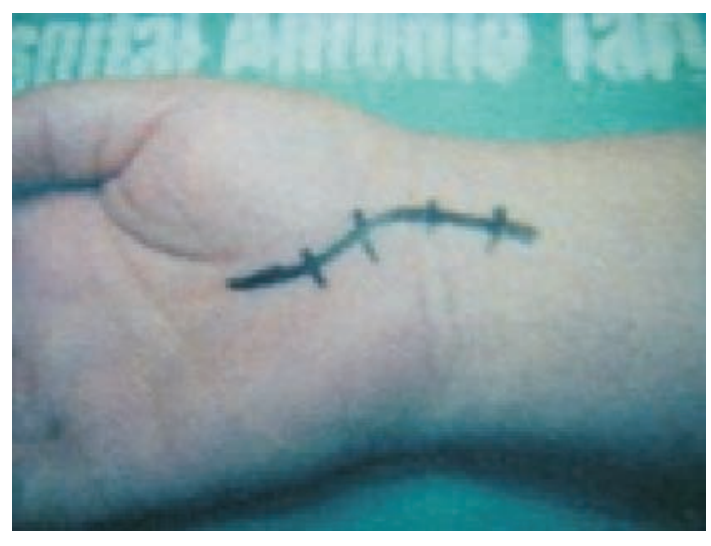

Figura 1 - Incisão convencional. 


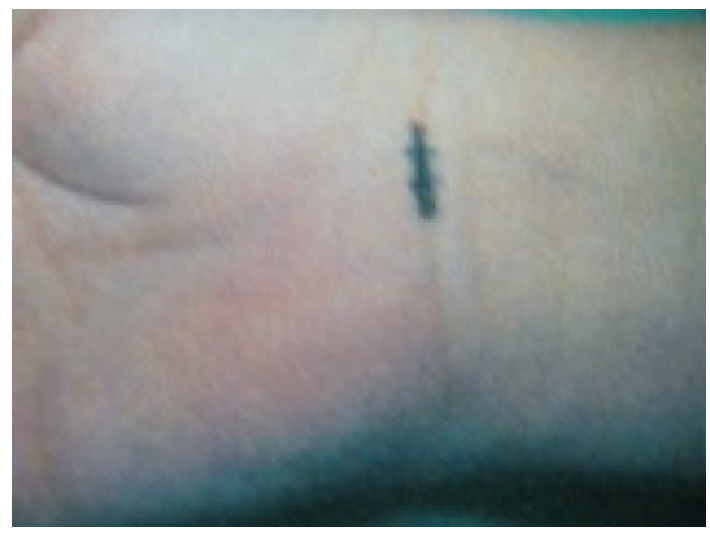

Figura 2-Microincisão.
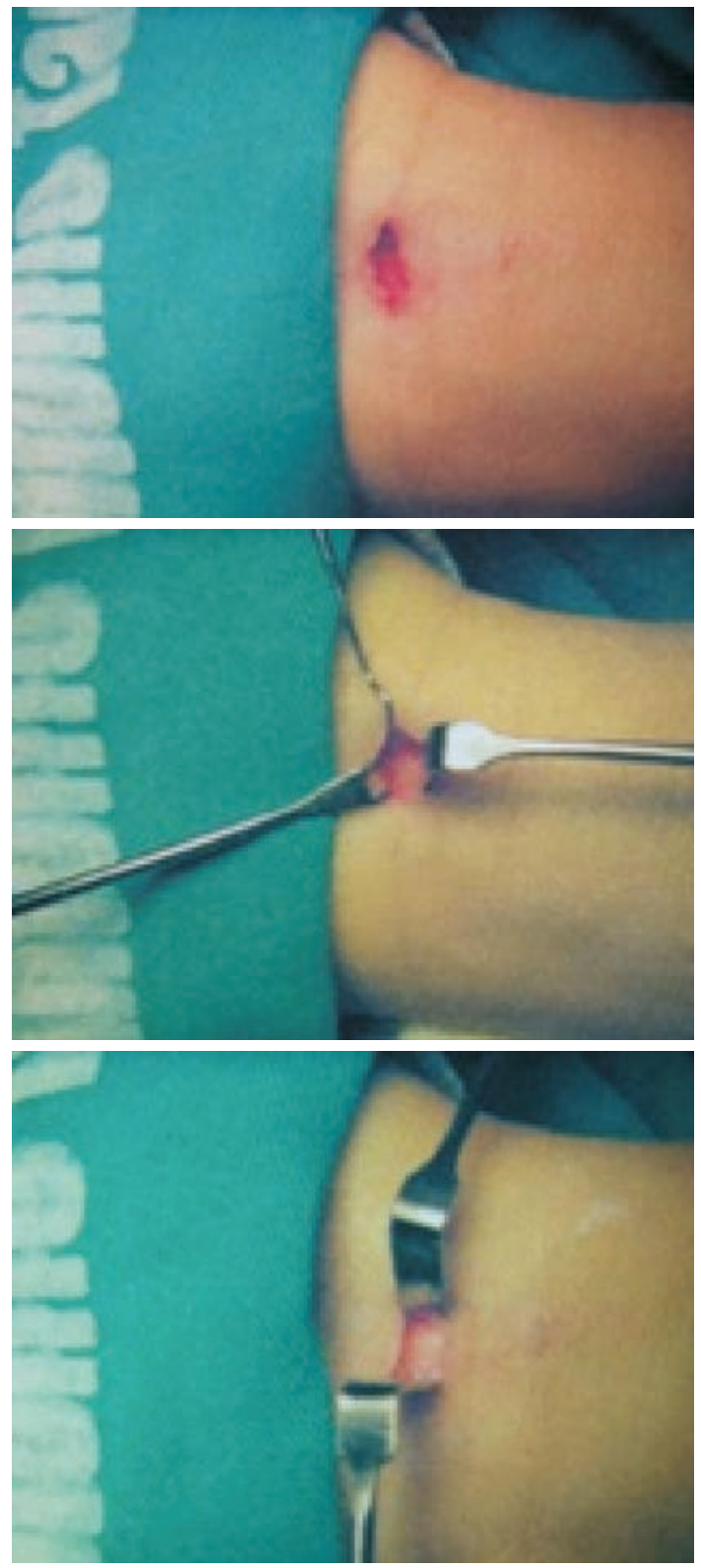

Figura 3 - Sequência cirúrgica.
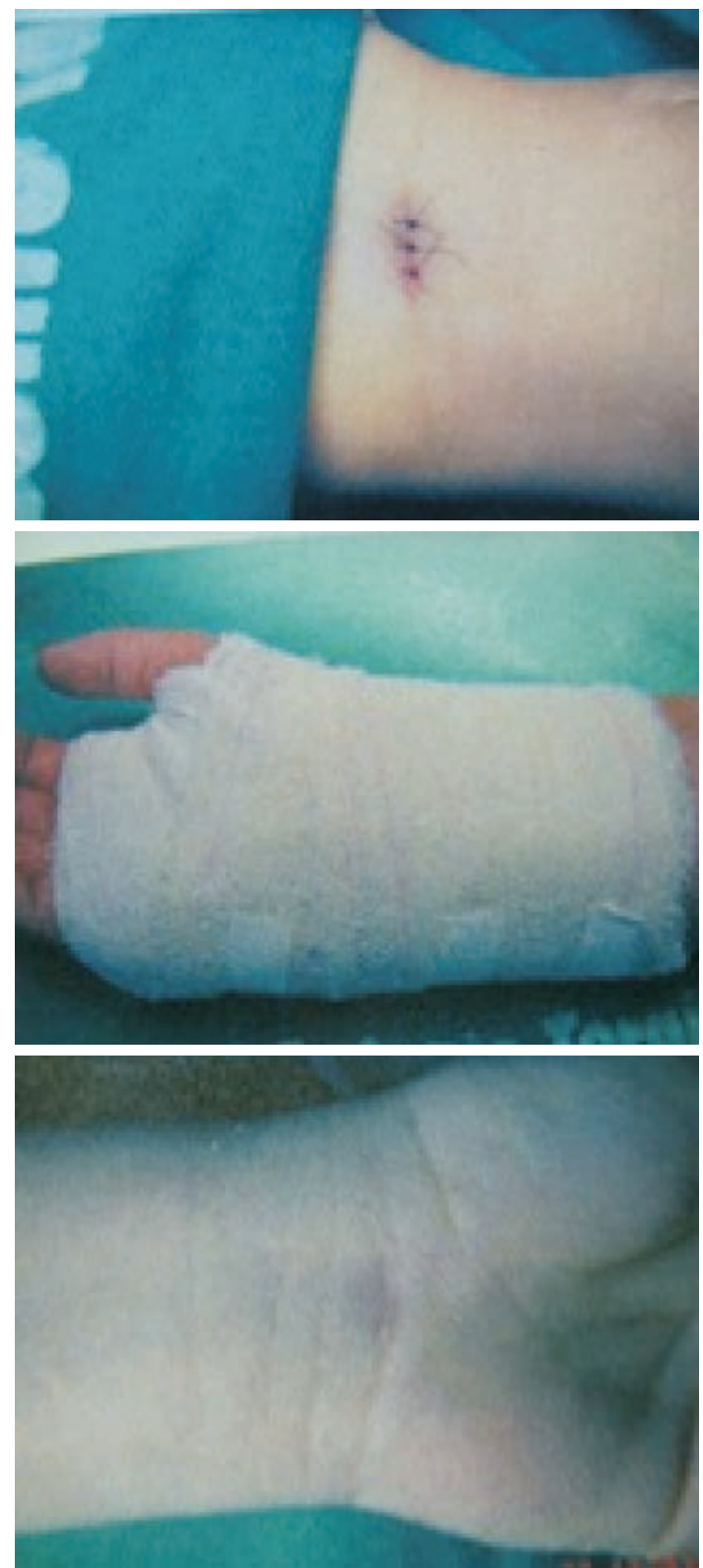

Figura 4-Pós-operatório sequencial.

\section{Discussão}

O ser humano é o alvitre de três características essenciais: um grande cérebro, a postura ereta e as mãos manipulativas. ${ }^{1}$ A mão tornou-se o fundamental utensílio por meio do qual o homem atua e modifica o mundo à sua volta. Para desempenhar esse papel, a mão apresenta uma estrutura abstrusa, responsável por prestezas que exigem exatidão e fazem com que ela esteja sujeita a lesões que podem levar à inépcia funcional. A STC, descrita pela primeira vez por Sir James Paget, ${ }^{2}$ em 1854, é, dentre as lesões que podem atingir 
o membro superior, a neuropatia de maior incidência. Ela versa na compressão do nervo mediano quando passa pelo túnel do carpo. ${ }^{3-5}$

Entre os fatores abarcados na fisiopatologia da síndrome, apesar de não haver consonância, destacam-se: sexo feminino, obesidade, índice de massa corporal alto, idade acima de 30 anos, atividade motora repetitiva (correlação não completamente estabelecida) e algumas doenças sistêmicas. ${ }^{4,17}$ Entre as causas clínicas citadas podem ser encontradas alterações que explicam o aumento de volume das estruturas presentes no interior do túnel do carpo, o qual é responsável pela constrição nervosa. ${ }^{9}$ O processo de instalação da STC por trabalho repetitivo pode ocorrer em decorrência de lesões agudas e crônicas no sistema musculoesquelético. A lesão aguda pode ser traumática ou por instalação de fadiga muscular, que acontece quando o nível de força aplicada é baixo, mas considerado acima da capacidade adaptativa do sistema muscular. As lesões crônicas são consequências de sobrecargas musculoesqueléticas a longo prazo. ${ }^{20}$

O quadro clínico é caracterizado por parestesias progressivas na mão, no início noturnas e, posteriormente, com duração mais prolongada, que submergem com movimentos da mão. As parestesias são localizadas na palma da mão e nos dedos, mas o paciente pode relatar sintomas dolorosos mais proximais, às vezes atingindo até o ombro. ${ }^{7,23} \mathrm{Com}$ a progressão da doença pode ocorrer fraqueza e atrofia dos músculos da eminência tenar. A pesquisa da sensibilidade pode estar alterada. Para se fazer o diagnóstico dessa síndrome, deve-se lançar mão do exame físico: teste de Phalen, teste de Tinel-Hoffmann, compressão do nervo mediano e sinal de fraqueza do músculo abdutor curto do polegar. ${ }^{13}$ Esses sinais não parecem ser muito precisos, embora sejam aceitos para detecção da STC, sendo recomendado seu uso, exceto dos testes de compressão do nervo mediano e Tinel-Hoffmann, os quais têm boa especificidade, mas pobre sensibilidade. ${ }^{13}$ Em outras palavras, esses testes determinam alto número de resultados falso-positivos e falso-negativos, o que os torna limitados como sinais clínicos $^{5,7,10-12,18}$ e devem ser utilizados em associação ao sintoma de dor noturna e à eletroneuromiografia. Nesta, é verificada a velocidade de condução nervosa por eletrodos de inserção; é considerada padrão-ouro para o diagnóstico de $\mathrm{STC}^{4}$ e mostra a saúde fisiológica do nervo mediano através do túnel do carpo. Os parâmetros utilizados nessa avaliação são latência motora distal, latência sensitiva distal, latência sensitiva da palma até o punho, estimulação seriada por meio do punho, comparação de latências sensitivas entre o nervo mediano e o ulnar e entre o mediano e o radial; comparação de latências sensitivas entre o nervo mediano e ulnar no dedo anular e eletromiografia. ${ }^{18}$
O tratamento proposto para STC é dividido em conservador e cirúrgico. ${ }^{3} \mathrm{O}$ tratamento conservador versa em fisioterapia e uso de medicamentos. Durante a fisioterapia são realizadas cinesioterapia e eletroterapia, além de prescrição de órteses. Todas essas medidas devem estar associadas a alterações nas atividades de vida diária, bem como daquelas realizadas no ambiente de trabalho. A outra vertente do tratamento argumenta a favor do tratamento cirúrgico, realizado pela primeira vez em outubro de 1929 por James Learmonth, ${ }^{4}$ com a secção do ligamento transverso do carpo, técnica popularizada mais tarde por Frederick P. Moersch. ${ }^{16}$ A cirurgia é recomendada quando tratamentos prévios, abrangendo medicamentos e injeção de esteroides intratúnel, falham. Os critérios de inclusão são os seguintes: parestesia constante ou intermitente ao longo do trajeto do nervo mediano, teste de Phalen positivo, distúrbios sensoriais e condução nervosa anormal. ${ }^{9}$ Os critérios de exclusão são: fratura prévia e lesões duplas, como radiculopatia cervical e polineuropatia. ${ }^{25}$

A cirurgia para liberação do túnel do carpo é um assunto corrente na literatura, motivo de diversas publicações, principalmente a partir dos anos 1950. Algumas descrevem vários tipos de complicações ${ }^{21,22,24,26}$ e muitas outras relatam excelentes resultados e baixos índices

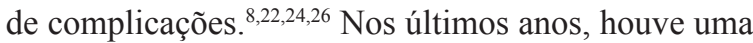
crescente utilização dos métodos endoscópicos para a liberação do túnel do carpo, com o objetivo de agilizar o retorno dos pacientes ao trabalho e diminuir a morbidade. A desvantagem dessa técnica é o elevado número de complicações operatórias e o alto custo do instrumental e do treinamento do cirurgião. . $^{6,19,22,24}$

Na liberação tradicional, o cirurgião faz uma incisão de $5 \mathrm{~cm}$ a $6 \mathrm{~cm}$, estendida longitudinalmente em relação à prega formada pelo punho, e realiza a liberação do ligamento transverso do carpo sob visualização direta. ${ }^{15}$ A liberação do ligamento também pode ser feita por via endoscópica, mas apresenta maior risco de lesionar o nervo mediano, porém o alívio dos sintomas é similar ao procedimento aberto, e os pacientes retornam ao trabalho mais cedo.

Sabedores dessas premissas previamente citadas, observamos com o passar do tempo que havia uma necessidade de melhorar a técnica cirúrgica do túnel do carpo, e por meios de conhecimentos anatômicos aliados a uma maior vivência com o assunto podemos por intermédio de análises desenvolver uma nova técnica que trará muitos benefícios ao paciente acometido por essa nosologia. A técnica cirúrgica desenvolvida por nós consiste em uma microincisão de $1 \mathrm{~cm}$ na linha média da prega do punho, levantamento da pele por meio de pequenos afastadores Farabeufs, avulsão dos planos até a completa visualização do retináculo dos flexores. Após a visualização, recortam-se os bordos do retináculo dos 
flexores, para evitar a recidiva da síndrome. Exatamente nessa nuance é que se encontra um dos grandes erros daqueles que executam esse procedimento; deixando as bordas do retináculo, quando cessar o edema das estruturas que estavam comprimidas pelo tendão, este voltará à posição prévia, ocorrerá fibrose e novamente angustiará o conteúdo do túnel do carpo, até mesmo o nervo mediano, trazendo à tona toda a sintomatologia com a mesma intensidade anterior à cirurgia, não se obtendo resultados definitivos para o paciente. Com nossa técnica, com base em nosso acompanhamento, praticamente inexiste a possibilidade de recidivas.

\section{Conclusão}

Os autores, por meio do acompanhamento desses pacientes, podem asseverar as vantagens atingidas por meio da técnica desenvolvida: são extraordinárias do ponto de vista clínico, diminuindo a chance de recidivas, trazendo melhor exequibilidade ao cirurgião, menor tempo cirúrgico e levando maior satisfação aos pacientes.

\section{Referências}

1. Agee JM, Mccarrol HR, Tortosa RD, Berry DA, Szabo RM, Peimer CA. Endoscopic release of the carpal tunnel: a randomized prospective multicenter study. J Hand Surg (Am). 1992:17:987-95.

2. Akalin EELO, Peker O, Senocak O, Tamci S, Gülbahar S, Çakmur R. Treatment of carpal tunnel syndrome with nerve and tendon gliding exercises. Am J Phys Med Rehabil. 2002;81:108-13.

3. Assunção AA, Almeida IM. Doenças ósteo-musculares relacionadas com o trabalho: membro superior e pescoço. In: Mendes, R. (Org.). Patologia do trabalho. Rio de Janeiro: Atheneu; 2002. p.1501-39.

4. Becker J, Nora DB, Gomes I, Stringari F, Seitensus R, Panosso JS. An evaluation of gender, obesity, age and diabetes mellitus as risk factors for carpal tunnel syndrome. Clin Neurophysiol. 2002;113:1429-34.

5. Berger AR, Herkovitz S. Cumulative trauma disorders. In: Rosenberg NL, editor. Ocupational and environment neurology. Boston: Butterworth-Heinemann; 1995. p.235-58.

6. Chow JC. Endoscopic release of the carpal ligament: a new technique for carpal tunnel syndrome. Arthroscopy. 1989;5:19-24.

7. Dawson DM. Entrapment neuropathies of the upper extremities. N Engl J Med. 1993;329:2013-8.

8. Gerritsen AA, de Vet HC, Scholten RJ, Bertelsmann FW, de Krom MC, Bouter LM. Splinting vs surgery in the treatment of carpal tunnel syndrome: a randomized controlled trial. JAMA. 2002;88:1245-51.

9. Karolczak APB, Vaz MA, Freitas CR, Merlo ARC. Síndrome do túnel do carpo. Rev Bras Fisioter. 2005;9:117-22.
10. Katz JN, Larson MG, Sabra A, Krarup C, Stirrat CR, Sethi $\mathrm{R}$, et al. The carpal tunnel syndrome: diagnostic utility of the history and physical examinations findings. Ann Intern Med. 1990;112:321-7.

11. Katz JN, Larson MG, Fossel AH, Liang MH. Validation of a surveillance case definition of carpal tunnel syndrome. Am J Publ Health. 1991;81:189-93.

12. Krom MCTFM, Kester ADM, Knipschild PG, Spaans F. Risk factors for carpal tunnel syndrome. Am J Epidemiol. 1990;132:1102-10.

13. Kuhlman KA, Hennessey WJ. Sensitivity and specificity of carpal tunnel syndrome signs. Am J Phys Med Rehabil. 1997;76:451-7.

14. Learmonth JR. The principle of decompression in the treatment of certain diseases of peripheral nerves. Surg Clin North Am. 1993;13:905-13.

15. Macdermid JC, Richards RS, Roth JH, Ross DC, King GJ. Endoscopic versus open carpal tunnel release: a randomized trial. J Hand Surg Am. 2003;28:475-80.

16. Moersch FP. Median thenar neuritis. Proc Staff Meet Mayo Clin. 1938;13:220-2.

17. Nordstrom DL, Vierkant RA, Albers JW, Becker MP, Armstrong TJ, Franzblau A. Risk factors for carpal tunnel syndrome in a general population. Occup Environ Med. 1997;54:734-40.

18. Oliveira JT. Síndrome do túnel do carpo: controvérsias a respeito de diagnóstico clínico e eletrofisiológico e a relação com o trabalho. Arq Neuropsiquiatr (Sao Paulo). 2000;58:1142-48.

19. Phalen GS. The carpal-tunnel syndrome. Seventeen years experience in diagnosis and treatment of six hundred fiftyfour hands. J Bone Joint Surg (Am). 1966; 48:211-28.

20. Sande LP, Coury JCG, Oishi J, Kumar S. Effect of musculoskeletal disorders on prehension strength. Appl Erg. 2001;32:609-16.

21. Scholten RJ, Gerritsen AA, Uitdehaag BM, van Geldere D, de Vet HC, Bouter LM. Surgical treatment options for carpal tunnel syndrome. Cochrane Database Syst Rev. CD003905, 2004.

22. Systematic review of randomized clinical trials of surgical treatment for carpal tunnel syndrome. $\mathrm{Br} \mathrm{J}$ Surg. 2001;88:1285-95.

23. Szabo RM, Slater RR, Farver TB, Stanton DB, Sharman WK. The value of diagnostic testing in carpal tunnel syndrome. J Hand Surg. 1999;24A:704-14.

24. Thoma A, Veltri K, Haines T, Duku E. A systematic review of reviews comparing the effectiveness of endoscopic and open carpal tunnel decompression. Plast Reconstr Surg. 2004;113:1184-91.

25. Uchiyama S, Toriumi H, Nakagawa H, Kamimura M, Ishigaki N, Miyasaka T. Postoperative nerve conduction changes after open and endoscopic carpal tunnel release. Clin Neurophysiol. 2002;113:64-70.

26. Zumiotti AV, Ohno PE, Prada FS, Azze RJ. Complicações do tratamento cirúrgico da síndrome do túnel do carpo. Rev Bras Ortop. 1996;31:199-202.

Original recebido em junho de 2009

Aceito para publicação em setembro de 2009

Endereço para correspondência

Luciano Ferreira de Holanda

Rua Delmiro Gouveia, 299

58107-735 - Campina Grande, PB

E-mail:luholanda@hat.com.br 